\title{
High Frequency Jet Ventilation during Initial Management, Stabilization, and Transport of Newborn Infants with Congenital Diaphragmatic Hernia: A Case Series
}

\author{
Qianshen Zhang, ${ }^{1,2}$ Jason Macartney, ${ }^{3}$ Lita Sampaio, ${ }^{4}$ and Karel O'Brien ${ }^{2}$ \\ ${ }^{1}$ Neonatal Intensive Care Unit, Maternal \& Child Healthcare Hospital of Shenzhen City, Guangdong 518028, China \\ ${ }^{2}$ Department of Paediatrics, Mount Sinai Hospital, University of Toronto, Toronto, ON, Canada \\ ${ }^{3}$ Department of Respiratory Therapy, The Hospital for Sick Children, Toronto, ON, Canada \\ ${ }^{4}$ Department of Respiratory Therapy, Mount Sinai Hospital, Toronto, ON, Canada
}

Correspondence should be addressed to Karel O’Brien; kobrien@mtsinai.on.ca

Received 8 July 2012; Accepted 19 December 2012

Academic Editor: Andrew Berry

Copyright (C) 2013 Qianshen Zhang et al. This is an open access article distributed under the Creative Commons Attribution License, which permits unrestricted use, distribution, and reproduction in any medium, provided the original work is properly cited.

\begin{abstract}
Objective. To review experience of the transport and stabilization of infants with CDH who were treated with high frequency jet ventilation (HFJV). Study Design. Retrospective chart review was performed of infants with antenatal diagnosis of CDH born between 2004 and 2009, at Mount Sinai Hospital Toronto, Ontario, Canada. Detailed information was abstracted from the charts of all infants who received HFJV. Results. Of the 55 infants, 25 were managed with HFJV at some point during resuscitation and stabilization prior to transport. HFJV was the initial ventilation mode in six cases and nineteen infants were placed on HFJV as rescue therapy. Blood gases procured from the umbilical artery before and/or after the initiation of HFJV. There was a significant difference detected for both $\mathrm{PaCO}_{2}(P=0.0002)$ and $\mathrm{pH}(P<0.0001)$. The pre- and posttransport vital signs remained stable and no transport related deaths or significant complications occurred. Conclusion. HFJV appears to be safe and effective providing high frequency rescue therapy for infants with $\mathrm{CDH}$ failing conventional mechanical ventilation. This paper supports the decision to utilize HFJV as it likely contributed to safe transport of many infants that would not otherwise have tolerated transport to a surgical centre.
\end{abstract}

\section{Introduction}

Congenital diaphragmatic hernia $(\mathrm{CDH})$ is one of the most challenging malformations that neonatologists and pediatric surgeons must manage $[1,2]$. In patients with antenatally diagnosed $\mathrm{CDH}$, the prognosis is dependent on both the degree of lung hypoplasia and persistent pulmonary hypertension (PPHN) after their birth $[1,3]$. The care of these infants has seen significant evolution, from previous aggressive ventilation and emergent surgical repair to current physiologic stabilization, standardized management protocols, gentle ventilation strategies, and delayed surgical repair, all in less than two decades [2].

Survival of patients with $\mathrm{CDH}$ is dependent on early diagnosis and improved resuscitation and transportation of an optimally-supported baby to a major surgical center for repair [3-5]. Infants with $\mathrm{CDH}$ may be diagnosed antenatally and deliver at a high risk perinatal center. Most infants with $\mathrm{CDH}$ require respiratory support with set limits on ventilatory pressures to avoid lung overdistension and acceptance of adequate rather than optimal $\mathrm{PaCO}_{2}$ and $\mathrm{PaO}_{2}$ [1]. High-frequency ventilation (HFV) allows gas exchange at low volumes thereby decreasing iatrogenic pulmonary barotrauma [6]. To date two modes of HFV has been studied in the care of infants with $\mathrm{CDH}$ : high frequency oscillatory ventilation (HFOV) and high frequency jet ventilation (HFJV). Careful use of HFV, either empirically or as rescue in infants requiring high peak inspiratory pressure (PIP) with conventional mechanical ventilation, appears to reduce mortality [7]. The use of HFV is well described in infants 
with $\mathrm{CDH}[8,9]$. However, transport with the HFO (3100A Sensormedics) is not an option as it does not have external battery power. HFJV is another mode of HFV which has previously been shown to be lung protective and can be used for transport as it has an external battery.

There is little published that describes the use HFJV during stabilization and transport of these infants [10]. The objective of this study was to review our site's experience with the transport and stabilization of infants with $\mathrm{CDH}$ who were treated with HFJV. Special considerations for managing an infant on HFJV during transport were also identified.

\section{Methods}

Following approval of the project by the Research Ethics Board of Mount Sinai Hospital, a retrospective chart review was performed of all infants with an antenatal diagnosis of $\mathrm{CDH}$ who were delivered at Mount Sinai Hospital, between January 01, 2004 and December 31, 2009. Mount Sinai Hospital $(\mathrm{MSH})$ is a perinatal referral centre for fetal anomalies in the province of Ontario, Canada, has a level 3 NICU and adjoins The Hospital for Sick Children (HSC), a pediatric surgical site, through an underground tunnel. The records of all liveborn infants with congenital diaphragmatic hernia were identified. Data was abstracted from records of the resuscitation, stabilization and transport of these infants in addition to the records at the surgical site. The parameters reviewed included the initial steps taken during resuscitation, initial ventilation settings, and acid-base balance. The criteria for starting HFJV, pharmacological support required, time and length of transport, and any complications that arose in transit were also reviewed.

Mount Sinai Hospital uses a standardized protocol for the resuscitation, and stabilization of these infants. Specifically, there are guidelines which limit the use of high airway pressures during conventional ventilation. Mean airway pressures $(\mathrm{MAP}) \leq 12 \mathrm{~cm} \mathrm{H}_{2} \mathrm{O}$ and PIP $\leq 25 \mathrm{~cm} \mathrm{H}_{2} \mathrm{O}$ are targeted. Also, preductal saturation $\geq 85 \%$ is accepted, as well as tolerating mild-to-moderate hypercarbia $\left(\mathrm{PaCO}_{2} \geq\right.$ $55 \mathrm{mmHg}, \mathrm{pH}>7.25)$. As this is considered a nonrecruitable lung disease, the use of HFV is recommended to optimize oxygenation while improving $\mathrm{CO}_{2}$ elimination. Nitric Oxide $(20 \mathrm{ppm})$ is initiated when the oxygen index is more than 20 or at the discretion of the attending physician, and $\mathrm{PGE}_{1}$ infusion or inotropic agents were started at the discretion of the clinical team. The transport team consisted of a fellow (paediatrician in training to be a neonatologist), one or two registered nurses, and two respiratory therapists.

An Airborne brand portable transport incubator (International Biomedical, USA) was used for all transports and modified to accommodate HFJV within this system. The Life Pulse high-frequency jet ventilator (Bunnell Inc, Salt Lake City, Utah) was used in tandem with a conventional ventilator to provide a source of heated, humidified bias flow. The iNOvent was used to deliver iNO which was blended into the constant flow of gas delivered through the HFJV circuit. The resuscitation team, under the direction of the attending neonatologist, determined the need to change from conventional mechanical ventilation to HFJV based
TABle 1: Patient characteristics.

\begin{tabular}{lc}
\hline Male $n(\%)$ & $16(64 \%)$ \\
Birth weight grams mean (sd) & $2868(820)$ \\
Gestational age weeks mean (sd) & $37.2(3.3)$ \\
Left sided $n(\%)$ & $19(78 \%)$ \\
Bilateral $n(\%)$ & $1(4 \%)$ \\
Congenital anomalies $n(\%)$ & $10(40 \%)$ \\
Preterm $n(\%)$ & $4(16 \%)$ \\
${ }^{*}$ O/E LHR mean (sd) & $39.3(17.2)$ \\
${ }^{* *}$ Probability for survival mean (sd) & $0.6(0.3)$ \\
\hline${ }^{*}$ Observed/Expected lung-to-head ratio. & \\
${ }^{* *}$ Predicted survival based CDH study Group predictive equation.
\end{tabular}

on guidelines for a protective lung strategy as presented above. Variables were recorded before and after changing the ventilator, shortly prior to departure from the referring hospital, and upon arrival and stabilization in surgical center. Data recorded as before and after were analyzed by paired $t$-test. Significance was defined as $P<0.05$.

\section{Results}

Fifty-five infants were born with antenatally diagnosed $\mathrm{CDH}$ during the 6-year-study period. HFJV was used at some point in the management of 25 of those infants, which is $45 \%$ of all the $\mathrm{CDH}$ births. Table 1 shows the data of demographics of $\mathrm{CDH}$ group. Sixteen were male and nine were female which corresponds to nearly a 2:1 male predominance. Ten infants (40\%) had congenital anomalies other than CDH, 9 (36\%) infants had known or suspected congenital heart disease, one with micrognathia and small kidneys, and one with bilateral pelviectasis also had a cardiac defect. Seven of the infants were born preterm (gestational age $<36+6$ weeks).

Table 1 also presents the data on predictors of survival, observed/expected lung-to-head ratio (O/E LHR), and the predicted survival rate based on the regression equation of the $\mathrm{CDH}$ study group. At the time of this study neither of these predictors was used to guide therapy in an individual patient.

The decision made to start a patient on high frequency ventilation was based on the clinical condition of the patient but in some patients was anticipated based on the severity of the infant's ultrasound findings. HFJV was the initial mode of ventilation used in six cases. In the remaining nineteen infants, HFJV was used as a rescue therapy for severe respiratory acidosis or hypoxemia despite maximal conventional ventilation parameters. One infant was transported on conventional ventilation because a provincial transport service was utilized that did not offer HFJV. The other infant was put back to conventional ventilation because of difficulties managing the infant on HFJV with secretions.

Seven of the 25 infants died in the delivery site prior to transport. Of the seven infants who died soon after birth, one had a bilateral $\mathrm{CDH}$ and cardiac disease, two had right-sided hernias (one with cardiac disease), and four had left-sided hernias (two with multiple anomalies and one with cardiac 


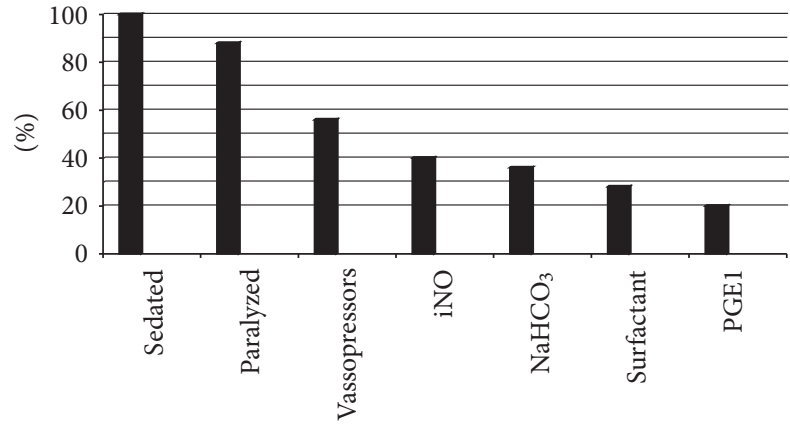

FIGURE 1: Management and interventions for all patient with $\mathrm{CDH}$ on HFJV.

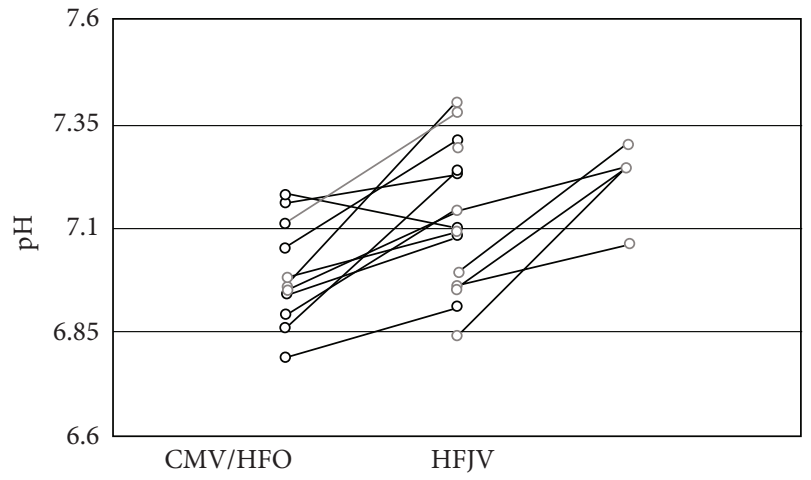

FIGURE 2: $\mathrm{pH}$ of patients before and after transition CMV or HFO to HFJV.

disease). The remaining 18 were transferred to HSC, two on conventional ventilation and 16 on HFJV.

Mount Sinai Hospital follows a protocol for managing $\mathrm{CDH}$ that defines failure of conventional ventilation as respiratory acidosis or hypoxemia despite PIP $>25 \mathrm{~cm} \mathrm{H}_{2} \mathrm{O}$ or MAP $>12 \mathrm{~cm} \mathrm{H}_{2} \mathrm{O}$. Two of the 25 infants developed a pneumomediastinum and four had pneumothoraces, three of whom died prior to transport.

Prior to transport all of the infants were sedated with either morphine or fentanyl and $88 \%(22 / 25)$ were paralyzed with Pavulon. Ten infants were transported on iNO. Seven received surfactant and five were treated with $\mathrm{PGE}_{1}$, seen in Figure 1.

Twenty one of the infants had at least two blood gases procured from the umbilical artery before and/or after the initiation of HFJV and were found to have significant improvement in blood gases. Figure 2 illustrates the $\mathrm{pH}$ changes pre- and postinitiation of HFJV for each infant. All but one infant showed an improvement in $\mathrm{pH}$; however, this infant actually experienced a decrease in $\mathrm{PaCO}_{2}$ and maintained a persistent metabolic acidosis.

For the comparison of pre- and postmeasurements of $\mathrm{PaCO}_{2}$ and $\mathrm{pH}$, a paired $t$-test was used (Figure 3). There was a significant difference detected for both $\mathrm{PaCO}_{2}(P=0.0002)$ and $\mathrm{pH}(P<0.0001)$.
The pre- and posttransport vital signs remained stable and no transport related deaths or significant patient complications occurred. The average length of transport from one site to the other was 30 minutes. The average time to rescue HFJV was 2 hours $14 \mathrm{~min}$. The median time of birth to time of arrival to HSC was 5 hours 20 min.

Data was collected from the Hospital for Sick Children on the sixteen patients transported using HFJV. Ten out of sixteen patients had the $\mathrm{CDH}$ surgically repaired, while care was withdrawn on six of the patients prior to surgery. Three of those infants had been treated with ECMO prior to their demise. The average age at time of surgery was $250.5 \mathrm{hrs}$ (10.44 days). Eight patients had patch repairs, one had a primary repair and one had a thorascopic repair. Out of the 10 surgically repaired patients, 3 did not survive to discharge. One had a recurrence of the $\mathrm{CDH}$ while on ECMO and did not undergo a second surgery. Care was withdrawn on the others on post-op day 13 and 18, respectively, because of respiratory failure. There were seven survivors of the 16 patients transported to HSC on HFJV. The average length of stay in the ICU prior to discharge was 37.14 days. The overall mortality for this entire series was therefore 64\% (16/25) despite maximal therapy including ECMO at the surgical site.

\section{Discussion}

In most patients with $\mathrm{CDH}$, respiratory management is complicated by the presence of multiple pathophysiologic challenges including hypoplastic and immature lungs and maldeveloped pulmonary vasculature [1-3]. The major advantage of high-frequency ventilation is improved oxygenation and ventilation through the use of small tidal volumes. Increased use of high-frequency ventilation, along with nitric oxide therapy, has decreased the need for ECMO in some centers [3-5]. HFJV has also been found to be effective in improving oxygenation and ventilation of neonates with $\mathrm{CDH}$ [11].

Our case series indicated that infants with $\mathrm{CDH}$ demonstrated significant improvement in ventilation upon initiation of HFJV and were able to be safely transported to a surgical hospital. These findings corroborate previous reports on the usefulness of HFJV as a rescue ventilation mode for neonates with respiratory failure supported by CMV $[12,13]$. The first, Boros et al., describes the use of HFJV on five patients reporting one survivor. While, Kuluz et al., comments on the use of HFJV as rescue therapy in 16 patients with a predicted survival rate of $63 \%$. The reported survival to discharge was $87 \%$, supporting the possible usefulness of this therapy.

Unlike most studies, this case series includes data on all infants with $\mathrm{CDH}$ without exclusion for LBW, prematurity, or congenital anomalies, yet they too experienced significant improvement in ventilation. Many different predictors of survival have been reported including the $\mathrm{O} / \mathrm{E}$ lung to head ratio and the $\mathrm{CDH}$ study group predictive equation. Both have been validated in infants with isolated $\mathrm{CDH}[14,15]$. The predicted survival of this case series using either the $\mathrm{O} / \mathrm{E}$ LHR or the $\mathrm{CDH}$ study group predictive equation was approximately $60 \%$ but our survival was $36 \%$. The fact that 

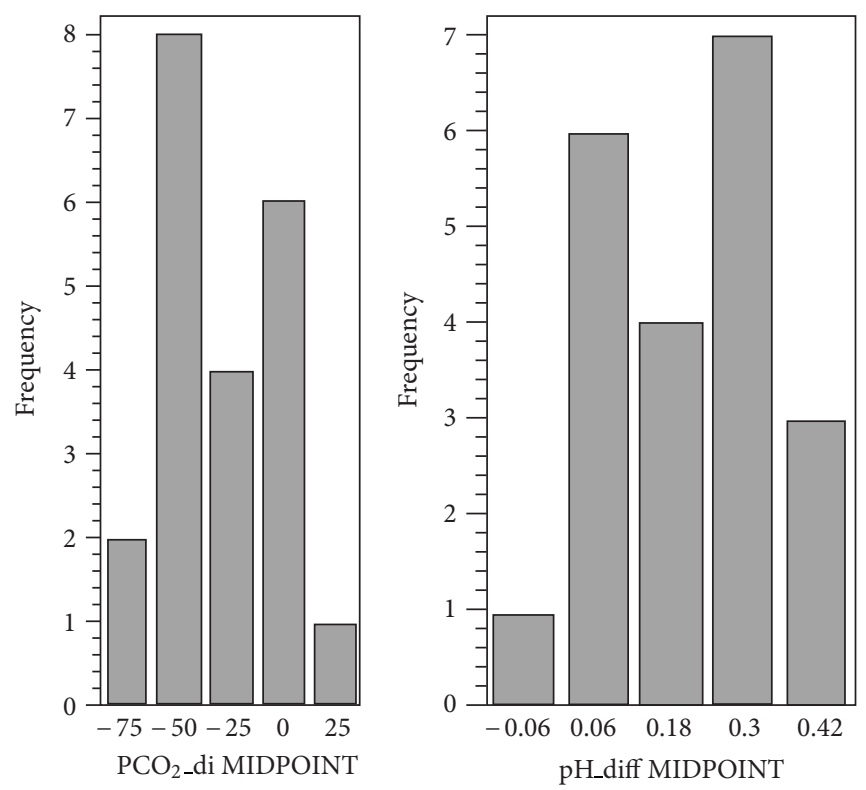

FIGURE 3: For the comparison of pre- versus post- $\mathrm{PaCO}_{2}$ and $\mathrm{pH}$ measurements, paired $t$-test was used. There was significant difference detected for both $\mathrm{PCO}_{2}(P=0.0002)$ and $\mathrm{pH}(P<0.0001)$.

more than half the infants, even those transported to the surgical site, did not survive to discharge, speaks to the severity of the illness and confirms the belief that it is indeed the most compromised infants that require HFJV in order to get to a surgical centre.

Pneumothoraces dominated the complications in our study; however diagnostic imaging was performed after HFJV was initiated so it is difficult to assess the timing of the insult and whether it was related to the change in mode of ventilation. Indeed, the decision to change from CMV or HFOV to HFJV before transport was at times due to the recognition of the inability of CMV to achieve acceptable oxygenation and ventilation at lower pressures in the presence of an air leak. All of the cases of pneumothorax occurred prior to transport and were not a complication of the transport itself and none of the neonates with continuing air leak were unstable during transport.

The use of HFV is well described in infants with $\mathrm{CDH}$, however HFOV (3100A Sensormedics) cannot be used for transport due to lack of an external battery. Therefore, our center used HFJV to ventilate infants who require HFV in order to facilitate ground transport. The staff of MSH NICU have a high comfort level with this mode of ventilation and its use for transport. Our success in safely transporting this compromised population is due in part to this. Other centers unfamiliar or inexperienced with HFJV certainly would not achieve the same results. More recently other forms of HFOV are available that can be used in transport. A randomized controlled multicenter trial may be warranted to clarify the role of HFJV versus HFOV on the long-term outcomes of $\mathrm{CDH}$.

Since this study is a retrospective analysis, there was no randomized assignment of ventilators (CMV or HFJV). This potential selection bias was mitigated by the fact that the sickest infants were placed on HFJV and yet it was these neonates that experienced a marked improvement. Despite the evidence of short-term improvement the overall benefit and long-term morbidity of this therapy needs to be further studied. To that end we are currently reviewing the overall outcomes of all antenatally diagnosed $\mathrm{CDH}$ at our sites. We also acknowledge the limited ability of our retrospective study to discuss minor or rare complications or adverse events during transport.

\section{Conclusions}

HFJV appears to be a safe and effective method of providing high frequency rescue therapy for infants with $\mathrm{CDH}$ failing conventional mechanical ventilation. Our data indicates that HFJV may be the preferred method of support for this subset of transported neonates due to its ability to optimize ventilation. HFJV can also be provided safely and efficaciously during transport. This paper supports the decision to utilize HFJV as it contributed to the safe transport of infants that may not otherwise have tolerated transport to a surgical centre. Clearly, a prospective randomized controlled, multicenter study would be needed to affirm these findings.

\section{Disclosure}

Dr. Q. Zhang, Dr. K. O’Brien, Mr. J. Macartney and Ms. L. Sampaio have disclosed no financial relationships relevant to this paper. This paper does not contain a discussion of an unapproved/investigative use of a commercial product/device. The study was approved by the Research Ethics Board of Mount Sinai Hospital, Toronto, Ontario, Canada. 


\section{Authors' contributions}

Dr. Q. Zhang is the first author who contributed to the design and acquisition of data, drafting, and revising the paper. Dr. $\mathrm{K}$. O'Brien is the Corresponding author and the supervisor of the research. She designed the study and revised the paper critically for important intellectual content. Mr. J. Macartney and Ms. L. Sampaio contributed to design and acquisition of data, analysis, and interpretation of data.

\section{Acknowledgments}

Special acknowledgments to Woojin Yoon, Micare center of Canadian Neonatal Network for the statistic help.

\section{References}

[1] K. S. Hartnett, "Congenital diaphragmatic hernia: advanced physiology and care concepts," Advances in Neonatal Care, vol. 8, no. 2, pp. 107-115, 2008.

[2] E. T. Tracy, S. E. Mears, P. B. Smith et al., "Protocolized approach to the management of congenital diaphragmatic hernia: benefits of reducing variability in care," The Journal of Pediatric Surgery, vol. 45, no. 6, pp. 1343-1348, 2010.

[3] L. Migliazza, C. Bellan, D. Alberti, A. Auriemma, G. Burgio, and G. L. E. A. Colombo, "Retrospective study of 111 cases of congenital diaphragmatic hernia treated with early highfrequency oscillatory ventilation and presurgical stabilization," The Journal of Pediatric Surgery, vol. 42, no. 9, pp. 1526-1532, 2007.

[4] J. T. Wung, R. Sahni, S. T. Moffitt, E. Lipsitz, and C. J. H. Stolar, "Congenital diaphragmatic hernia: survival treated with very delayed surgery, spontaneous respiration, and no chest tube," The Journal of Pediatric Surgery, vol. 30, no. 3, pp. 406-409, 1995.

[5] R. A. Cortes, R. L. Keller, T. Townsend et al., "Survival of severe congenital diaphragmatic hernia has morbid consequences," The Journal of Pediatric Surgery, vol. 40, no. 1, pp. 36-45, 2005.

[6] J. W. Logan, C. M. Cotton, R. N. Goldberg et al., "Mechanical ventilation strategies in the management of congenital diaphragmatic hernia," Seminars in Pediatric Surgery, vol. 16, no. 2, pp. 115-125, 2007.

[7] M. Keszler and D. J. Durand, "Neonatal high-frequency ventilation. Past, present and future," Clinics in Perinatology, vol. 28, no. 3, pp. 579-607, 2001.

[8] P. Van Reempts, C. Borstlap, S. Laroche, and J. C. Van der Auwera, "Early use of high frequency ventilation in the premature neonate," European Journal of Pediatrics, vol. 162, no. 4, pp. 219-226, 2003.

[9] R. F. Soll, "The clinical impact of high frequency ventilation: review of the Cochrane meta-analyses," Journal of Perinatology, vol. 26, supplement 1, pp. 38-42, 2006.

[10] E. S. Mainali, C. Greene, H. J. Rozycki, and G. R. Gutcher, "Safety and efficacy of high-frequency jet ventilation in neonatal transport," Journal of Perinatology, vol. 27, no. 10, pp. 609-613, 2007.

[11] M. A. Kuluz, P. B. Smith, S. P. Mears et al., "Preliminary observations of the use of high-frequency jet ventilation as rescue therapy in infants with congenital diaphragmatic hernia," The Journal of Pediatric Surgery, vol. 45, no. 4, pp. 698-702, 2010.
[12] M. Keszler, S. M. Donn, R. L. Bucciarelli et al., "Multi-center controlled Trial comparing high-frequency jet ventilation and conventional Mechanical ventilation in new born infants with pulmonary interstitial emphysema," The Journal of Pediatrics, vol. 119, no. 1, part 1, pp. 85-93, 1991.

[13] M. Keszler, H. D. Modanlou, D. S. Brudno et al., "Multicenter controlled clinical trial of high-frequency jet ventilation in preterm infants with uncomplicated respiratory distress syndrome," Pediatrics, vol. 100, no. 4, pp. 593-599, 1997.

[14] The Congenital Diaphragmatic Hernia Study Group, "Estimating disease severity of congenital diaphragmatic hernia in the first 5 minutes of life," The Journal of Pediatric Surgery, vol. 36, no. 1, pp. 141-145, 2001.

[15] M. A. Alfaraj, P. S. Shah, D. Bohn et al., "Congenital diaphragmatic hernia: lung-to-head ratio and lung volume for prediction of outcome," American Journal of Obstetrics and Gynecology, vol. 205, no. 1, pp. 43.e1-43.e8, 2011. 


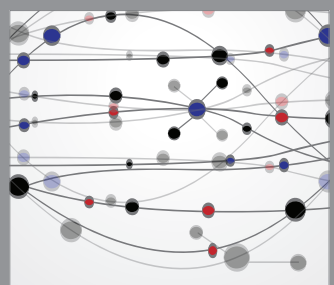

The Scientific World Journal
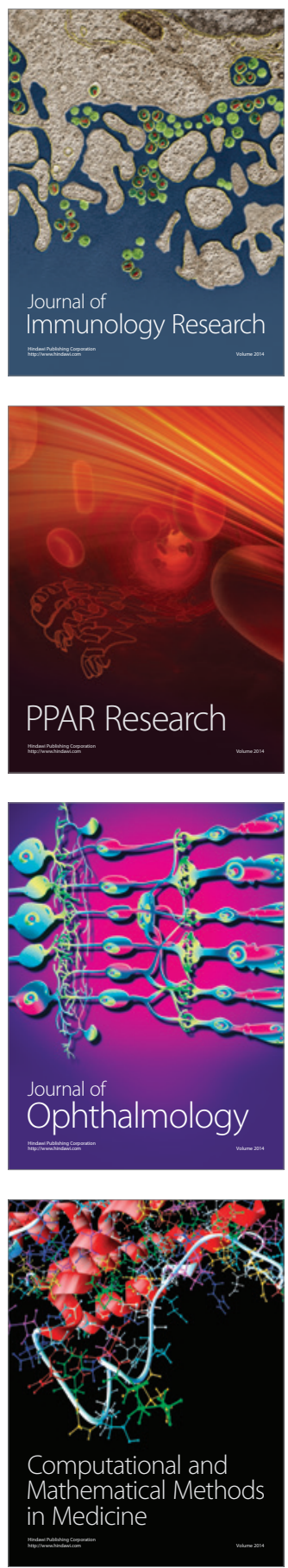

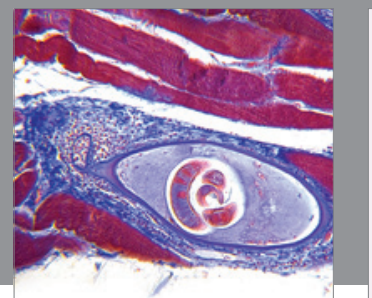

Gastroenterology

Research and Practice
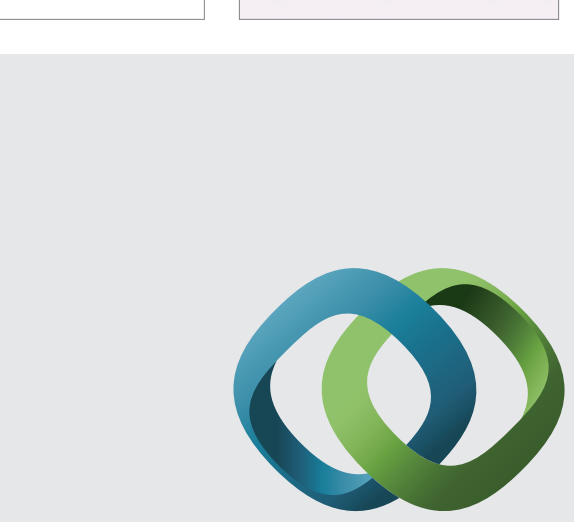

\section{Hindawi}

Submit your manuscripts at

http://www.hindawi.com
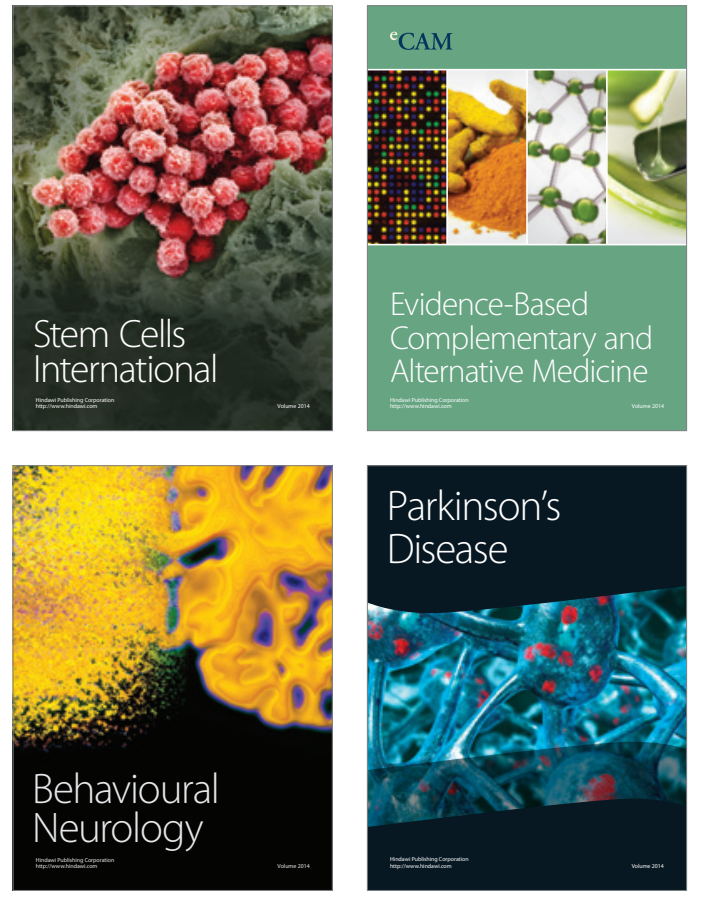
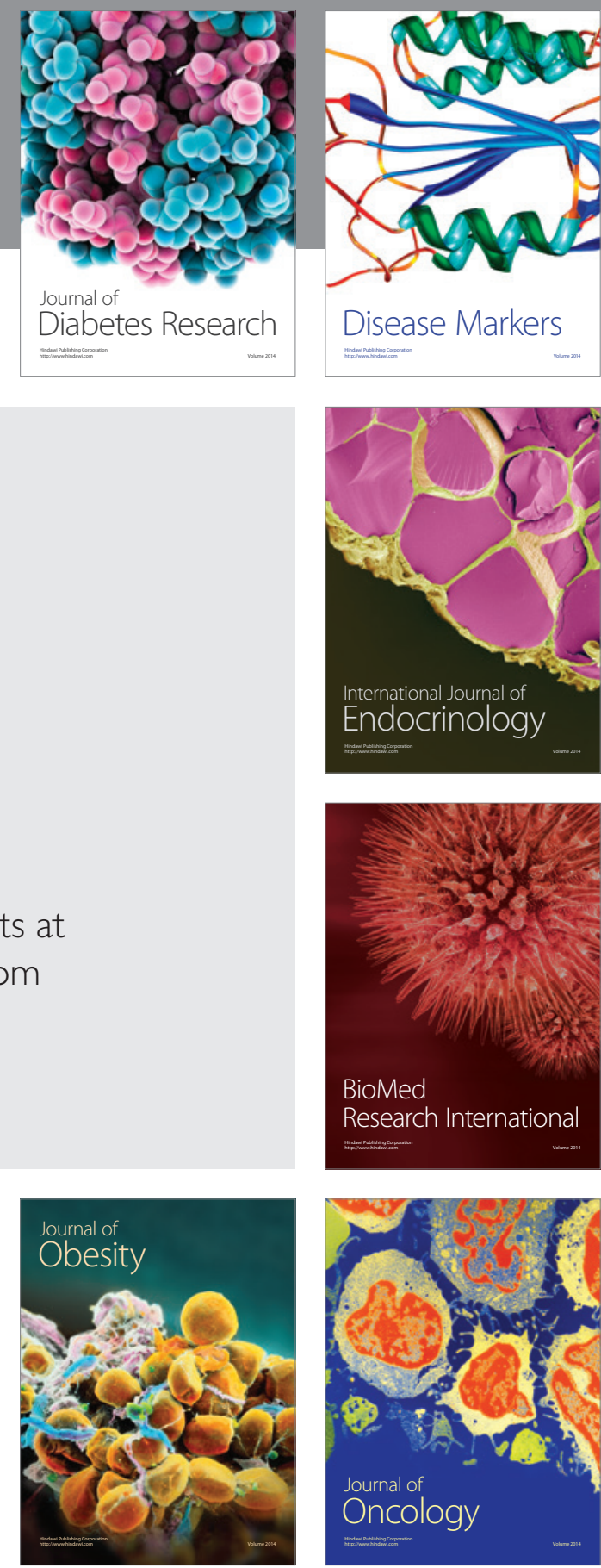

Disease Markers
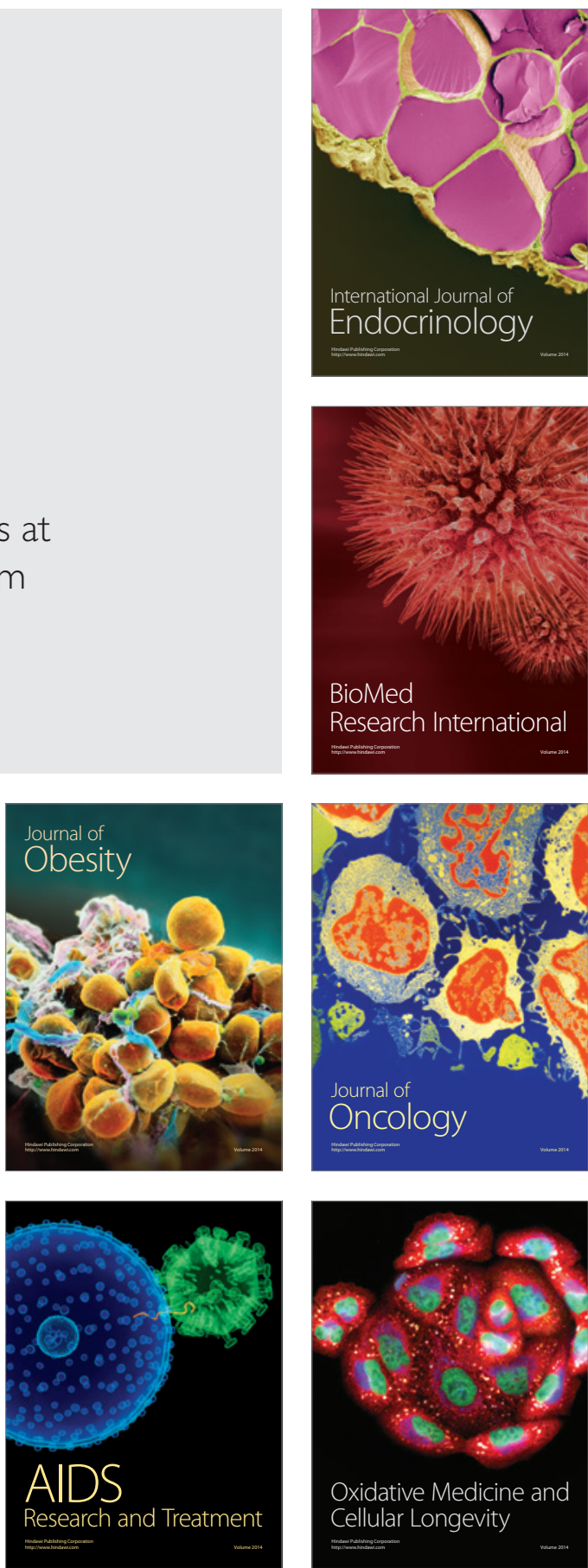